\title{
A combinatorial classic - sparse graphs with high chromatic number
}

\author{
Jaroslav Nešetřil* \\ Computer Sience Institute of Charles University \\ and \\ Institute for Theoretical Computer Science (ITI) \\ Charles University \\ Malostranské nám.25, 11800 Praha 1 \\ Czech Republic \\ Email: nesetril@iuuk.mff.cuni.cz
}

Remembering dědeček Paul Erdős

\section{Introduction}

It seems that combinatorics, and graph theory in particular, reached mathematical maturity relatively recently. Perhaps as a result of this there are not too many essential stories which have determined the course of the subject over a long period, enduring stories which appear again and again as a source of inspiration and motivate and challenge research.

In this article we attempt to demonstrate one example of such a story which we believe motivated some of the key parts of modern combinatorics. (Of course there are other stories, see for example [59].) Moreover the main result is related to the central theme of this book - the work and mathematical legacy of Paul Erdős.

* Partially supported by the Project LL1201 ERCCZ CORES and by CE-ITI P202/12/G061 of GACR. 
Let $G=(V, E)$ be an (undirected) graph. We need to recall only a few facts and definitions. The chromatic number $\chi(G)$ of $G$ is the minimal number of classes ("colors") of a partition of $V$ into independent sets. A set $A \subseteq V$ is called independent if it doesn't contain any edge. The maximal size of an independent set is denoted by $\alpha(G)$. It is obvious that

$$
\alpha(G) \cdot \chi(G) \geq|V|
$$

holds for every graph $G$.

This leads to the lower bound

$$
\chi(G) \geq \frac{|V|}{\alpha(G)},
$$

which is one of the very few lower bounds available for the chromatic number.

It is a classical (and folklore) result that a graph has chromatic number $\leq 2$ iff it doesn't contain a cycle of odd length. An even easier statement is that a forest (i.e. a graph without any cycle) has chromatic number $\leq 2$.

The minimal length of a cycle in $G$ is called the girth of $G$ and denoted here by $\operatorname{girth}(G)$. The central result of this paper has the following innocent form:

Theorem 1 (Erdös [23]) For every choice of positive integers $k$ and $l$ there exists a graph $G_{k, l}=G$ with the following properties:

1) $\chi(G) \geq k$;

2) $\operatorname{girth}(G)>l$.

Thus the absence of a short cycle (of length $\leq l$ ) cannot guarantee bounded chromatic number. By interpreting the chromatic number as a dimension or as a measure of complexity we see that Theorem 1 claims that there exists high dimensional (or highly complex) graphs which are locally as trivial as forests (i.e. graphs without any cycles) can be. An old saying existing in several languages is very fitting here: We do not see the wood for the trees! Yes, these are paradoxical objects.

Theorem 1 is both a culmination of long development and the start of important consequent research and methods. The literature is large and we find Theorem 1 in most books dealing with graphs. With various proofs one can find it in many combinatorial graph theory books and particularly in 
books relating to probabilistic methods in combinatorics, see e.g. [11], [5], [39], [13], [21], [63], [10], [57]. In this survey we concentrate on various structural extensions and theoretical implications of Theorem 1 (and we indicate various proof methods).

\section{Early constructions}

Theorem 1 was proved in 1958 by Erdős in his seminal paper [23]. But already at that time this result was firmly based in advanced combinatorics and it also had an interesting history. Let us review it briefly for completeness from a contemporary perspective.

The first nontrivial instance of Theorem 1 is the case $l=3$. In this form claims the existence of a triangle free graph $G_{k, 3}$ with $\chi\left(G_{k, 3}\right)>k$. This was proved independently by W. Tutte (alias Blanche Descartes) [20] and A. Zykov [92]. The proofs are constructive and can be visualised as follows:

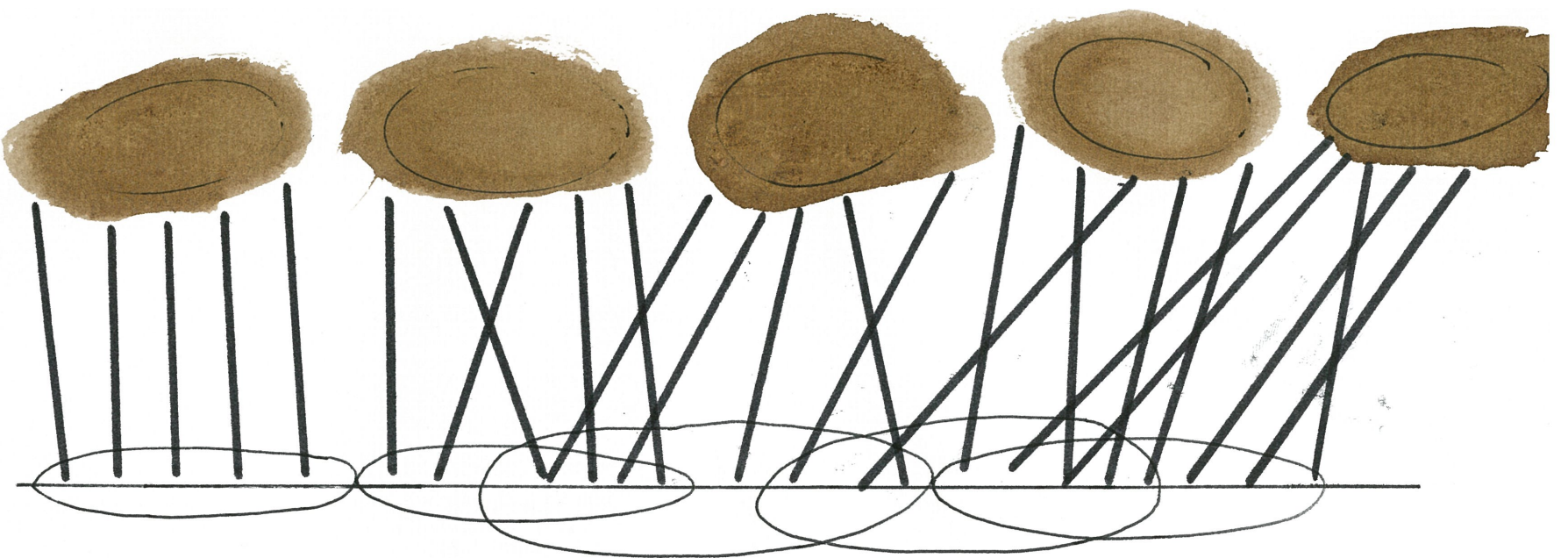

Here is a more formal sketch: We proceede by induction on $k$. Given $G=G_{k, 3}$ with $n$ vertices we consider a set $X$ with $k(n-1)+1$ vertices and for every subset $Y \subseteq X,|Y|=n$ we take an isomorphic copy $G_{Y}$ of $G$ every vertex of which is joined by a matching $E_{Y}$ to $Y$. Denote by $G_{k+1,3}$ the graph consisting of all edges in all graphs $G_{Y}, Y \subseteq X,|Y|=n$, and all matchings $E_{Y}$. It is easy to see that $G_{k+1,3}$ has no triangles and, assuming $\chi\left(G_{k, 2}\right) \geq k$, we get $\chi\left(G_{k+1,3}\right) \geq k+1$.

Tutte's construction is a prototype of many subsequent proofs and variants, as we shall see in Sections 4 and 5 . Let us give some further constructions of triangle-free graphs (i.e. $l=3$ ), most of which are regarded as classical. 
Note that already in [43] it was observed that the above inductive construction does not even create cycles of length $\leq 5$. However this remained the best result (with respect to girth $l$ ) until [23].

Another early construction for $l=3$ was provided by [66]. The construction proceeds again by induction on $k$ : In each step we create a sibling $x^{\prime}$ for every vertex $x$ and join $x^{\prime}$ to a vertex $y$ if and only if $x$ and $y$ are joined. Then we add a (universal) vertex joined to all the siblings vertices produced. Call the resulting graph $M(G)$ (Mycielskian of $G$ ). $M(G)$ has no triangle and $\chi(M(G))=\chi(G)+1$. (Thus from $K_{2}$ we obtain $C_{5}$ and from $C_{5}$ the Grötzsch graph.)

An interesting variation of this construction of graphs $G_{k, 3}$ is to iterate siblings. By this we mean that every vertex has siblings $x_{1}, \ldots, x_{t}$ and sibling $x_{i+1}$ is joined to those siblings $y$ for which $\{x, y\} \in E$. A universal vertex is then joined to all siblings $x_{t}$. These graph (and their variants) were studied in [32], [87], [8].

One of the simplest constructions is provided by the shift graphs $S_{n}$ : the vertices of $S_{n}$ are all pairs $(a, b)$ of integers $1 \leq a<b \leq n$ with edges formed by pairs $(a, b)(b, c)$. Clearly $S_{n}$ has no triangle (but contains large complete bipartite graphs) and $\chi\left(S_{n}\right)=\lceil\log n\rceil$. These remarkable graphs are important in the infinite case as well and they can be traced to ErdösSpecker graphs [28].

Other early constructions of triangle-free graphs with high chromatic number are geometrical (distance graphs, see already [27]). A particularly elegant combinatorial geometric construction [19] was discovered in the context of computational complexity:

We consider the set of all flags (i.e. all incidence pairs $(p, L))$ in a projective plane of order $k$ with an arbitrary linear ordering $<$. These are the vertices of our graph $G$. Vertices $(p, L)$ and $\left(p^{\prime}, L^{\prime}\right)$ will form an edge of $G$ if $(p, L)<\left(p^{\prime}, L^{\prime}\right)$, all $p, L, p^{\prime}, L^{\prime}$ are distinct, and if $p \in L^{\prime}$. This graph has no $K_{3}$, and it can be shown that $\alpha(G) \leq k+1$. Thus $\chi(G) \geq k^{2}+k+1$ as $G$ has $\left(k^{2}+k+1\right)(k+1)$ vertices.

Another by now classical example is provided by Kneser graphs. The Kneser graph $K\left(\begin{array}{l}n \\ p\end{array}\right)$ has as vertices all $p$-element subsets of $[n]=\{1,2, \ldots, n\}$. Edges of $K\left(\begin{array}{l}n \\ p\end{array}\right)$ are formed by pairs of disjoint sets. In (another) landmark paper [56] Lovász proved that $\chi\left(K\left(\begin{array}{l}n \\ p\end{array}\right)\right)=n-2 p+2$. This (lower bound) was achieved by relating the coloring problem to algebraic topology. This powerful tool found many applications (see Matoušek's book [61] devoted to 
this subject). This is clearly an "advanced" construction (with which we deal in the next section) but it is related to girth 4 only. It follows that the Kneser graphs $K\left(\begin{array}{c}2 m+k-2 \\ m\end{array}\right)$ (for any $m$ ) provide another nice example in playing the role of $G_{k, 3}$.

All these constructions have been thoroughly studied. Any new construction (such as [19] or [49]) is welcome with high hopes and then investigated thoroughly (see e.g. [8], [46] [32], [87]). But all these old-new constructions, which we have not listed exhaustively, are related to small girth. Indeed very small: $l \leq 6$ and mostly even $l=3$. One should stress that the odd girth condition (i.e. the absence of short odd cycles) is in the context of chromatic number a much easier condition than the girth. Rectangles present a problem and there are structural reasons for it (see more on that in the last section).

\section{Erdős theme}

Theorem 1 for $l=3$ (i.e. the existence of triangle-free graphs with large chromatic number) provided only one part of the motivation for Erdös' proof for general $l$. The other motivation (and certainly at that time for Erdös more important motivation) was the setting which relies on the inequality (2) and relates to Ramsey theory [30]. It is interesting to follow [23]:

Denote by $r(k, 3)$ the minimal number of vertices $n$ such that every triangle-free graph $G_{n}$ with $n$ vertices contains an independent set of size $k$. Formally,

$$
r(k, 3)=\min \left\{n \text {; either } K_{3} \subseteq G_{n} \text { or } \alpha\left(G_{n}\right) \geq k\right\} .
$$

Erdös proved in [23] that $r(k, 3)>k^{1+1 / 6}$, which using (2) implies that there are graphs $G_{k, 3}$. The asymptotic behavior of Ramsey numbers $r(k, 3)$ was determined in a sequence of important papers [44], [1],

$$
c_{1} \frac{k^{2}}{\log k} \leq r(k, 3) \leq c_{2} \frac{k^{2}}{\log k},
$$

and gave rise to Rödl's nibble, or semi-random methods [85], [5], [41]. The numbers $r(k, 3)$ were the first asymptotically known Ramsey numbers (since then there have been others, see [3], [4]).

Returning to the history of Theorem 1, Erdös made a stronger statement:

Theorem 2 ([23]) Let $l$ be fixed, let $0<\eta<\frac{1}{2 l}$. For every sufficiently large $n$ there exists a graph $G=(V, E)$ with $n$ vertices and the following properties: 

1) $\operatorname{girth}(G)>l$;
2) $\alpha(G)<n^{1-\eta}$.

(Note that this not only implies Theorem 1 but proves further that $\chi(G)>n^{\varepsilon}$.)

The proof of Theorem 2 is probabilistic and in fact it may be viewed as the cradle of the probabilistic method [88]. Nowadays it is found in every good graph theory book. Here is a very brief sketch:

We consider a random graph $G$ with $n$ vertices and $n^{1+\varepsilon}$ edges, $\varepsilon=2 \eta$, and prove that almost all such graphs satisfy 2) and that they contain $o(n)$ edges in cycles of length $\leq l$. They can then be deleted while 2) still holds.

Putting it more poetically: A thin soup of $n^{1+\varepsilon}$ edges on $n$ points contains (on average) few cycles but still shares some properties of the complete graph, namely small independence number.

How to depict this proof? Like a soup.

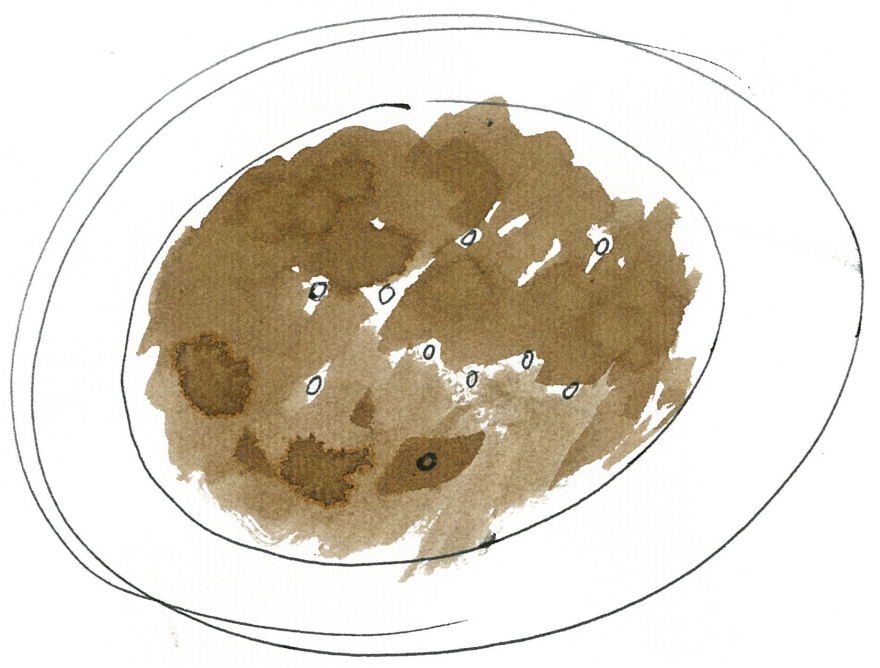

The proof of Theorem 2 has many variants and many more problems were solved by this method. Continuing in our line, for example, Erdős and Hajnal [25] generalized the result to hypergraphs as follows:

A $p$-uniform hypergraph is a pair $(X, \mathcal{M})$, where $\mathcal{M} \subseteq\left(\begin{array}{l}X \\ p\end{array}\right)=\{M ; M \subseteq$ $X,|M|=p\}$. Elements of $\mathcal{M}$ are still called edges. A cycle in $(X, \mathcal{M})$ and its length and girth are defined analogously as for graphs; a cycle of length 2 is formed by any pair of edges which intersect in (at least) 2 points. 


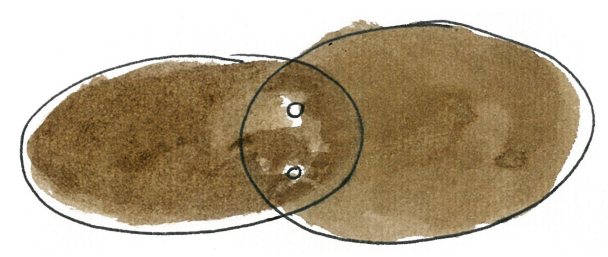

Hypergraphs without 2-cycles are called simple (or linear).

The chromatic number $\chi(X, \mathcal{M})$ is defined analogously as for graphs: it is the minimal number of colors needed in a coloring of vertices so that no edge is monochromatic (this seems to be the most common definition of the chromatic number for hypergraphs; of course there are other possibilities).

Theorem $3([\mathbf{2 5}])$ Let $p \geq 2, k, l$ be positive integers. Then there exists a p-uniform hypergraph $G_{p, k, l}=(X, \mathcal{M})$ such that

1) $\chi(X, \mathcal{M}) \geq k$;

2) $\operatorname{girth}(X, \mathcal{M})>l$.

The probabilistic proofs of Theorems 2 and 3 are similar. Yet the connection between these two statements provide some challenging open problems (as we shall stress at several places in this article).

\section{Advanced constructions}

Already in Erdös' paper [23] the question about a constructive proof of Theorem 1 is raised. The progress has been very slow here. The $l=6$ barrier was broken only a decade later [67] and there were speculations about the untractability (in some sense) of the problem. Even from today's point of view there is no easy (and elementarily justified) construction of a graph, say, $G_{4,27}$.

The first constructive proof of Theorem 1 was obtained by Lovász [55]. His striking proof is based on proving (the more general) Theorem 3, i.e. the existence of the hypergraph $G_{p, k, l}$. The proof proceeds by double induction on $p$ and $l$ (for a fixed $k$ ) and is too complicated to be explained here.

Another construction was provided in [77]. This is an outgrowth of structural Ramsey theory. It is called partite construction or amalgamation construction [80],[74], [75], [73] and in the structural Ramsey theory it seems to be one of the basic methods for obtaining structural results. The partite construction when applied to coloring of vertices is indicated by the following: 


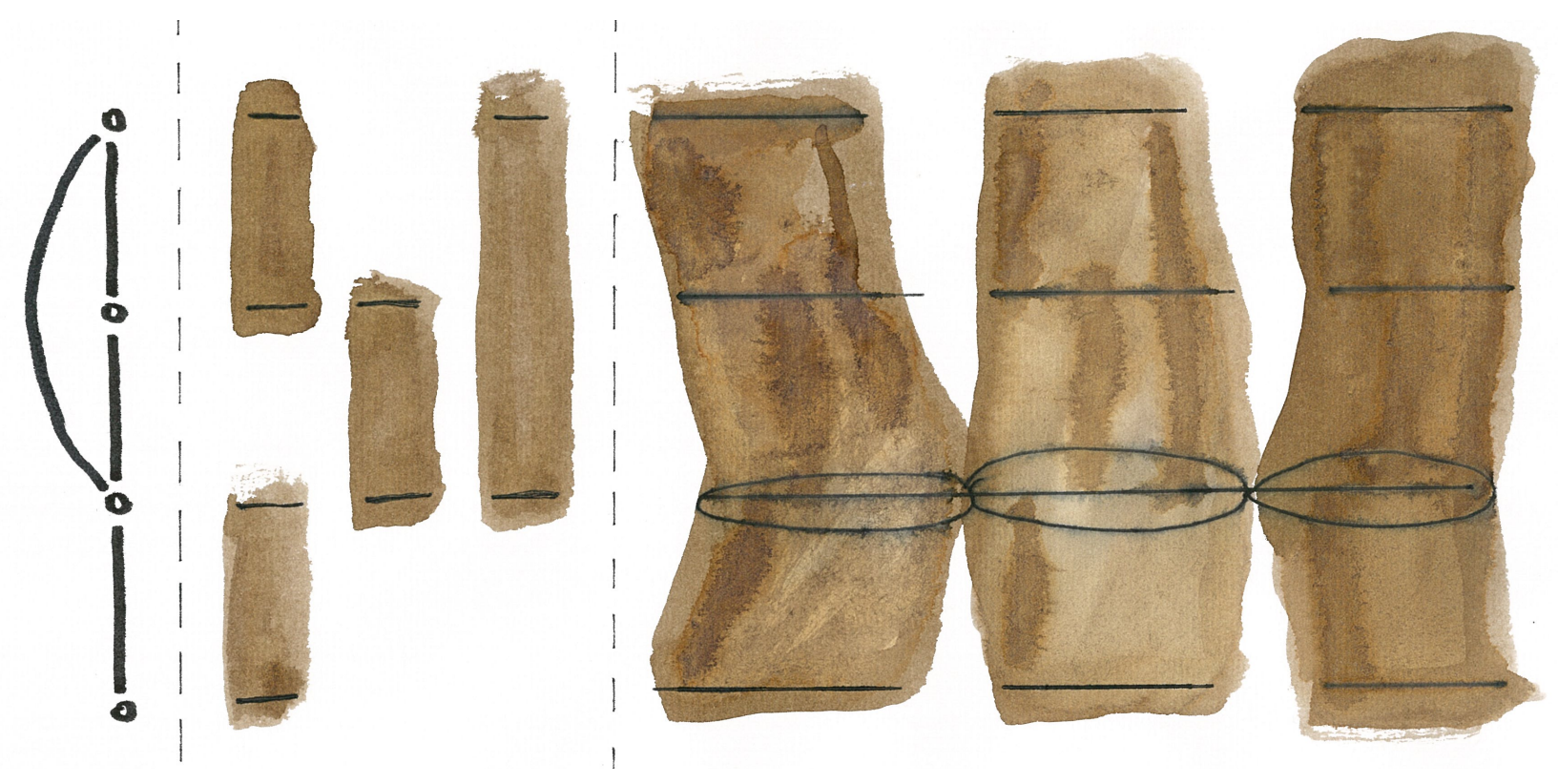

Here is a very rough sketch: Put $a=(p-1)(k-1)+1$. We start with a system $(V, \mathcal{M})$ of $p$-tuples which are organised on the set $V=\bigcup_{i=1}^{a} V_{i}$, (the $V_{i}$ are disjoint sets called parts), in such a way that for any $p$-tuple of parts $V_{i_{1}}, \ldots, V_{i_{p}}$ there exists an edge $M \in \mathcal{M}$ with $M \subseteq \bigcup_{j=1}^{p} V_{i_{j}}$. We call this the partite system $P_{0}$.

In the inductive step we assume that we are given a partite system $P_{i-1}=$ $(V, \mathcal{M})$ with $V=V_{1} \cup \ldots \cup V_{a}$. Put $\left|V_{i}\right|=P$ and apply induction to get system $(Y, \mathcal{N})$ with properties $(P, k, l-1)$. Now extend (like in Tutte's construction) every $N \in \mathcal{M}$ to a copy of $P_{i-1}$ while keeping the distribution to parts. One then proves (see [38] for more details) that $P_{a}$ has properties of $G_{p, k, l}$.

In a way the partite construction is a multipartite generalization of Tutte's construction. (The situation is not so straightforward for Ramsey theory and the amalgamation is more complicated.)

However for both Lovász's construction and as well as for the partite construction the size of the constructed (hyper)graph is not bounded by a tower function of bounded height. Even the (somewhat less precise) question whether one can prove Theorem 1 without referring to Theorem 3 was asked (and answered positively in [49]). Further variants of constructions of graphs $G_{k, l}$ are given in [50] and more recent [89].

One should stress that the size of the graphs $G_{k, l}$ is not merely a combinatorial question. The graphs $G_{k, l}$ are closely linked to special graphs used in the theory of algorithms and complexity theory. In particular, expander graphs (see for example extensive) [38] form a cornerstone of the modern 
theory of computing (see for example Ajtai-Komlos-Szemeredi [2]). One can see easily that large $d$-regular expander graphs with girth $l$ may be used to construct graphs $G_{k, l}$.

A polynomial size construction of expanders, and thereby of graphs $G_{k, l}$, came as a real surprise from a different corner of mathematics as a combination of mainly harmonic analysis, number theory and algebraic graph theory. The resulting graphs, often called Ramanujan graphs defined by Margulis [60] and Lubotzky, Phillips and Sarnak [58], are fascinating in their own right.

There is a large literature (an interested reader may consult a survey article [38] and references given there) and several books. For completeness we state the main consequence for the context of this paper:

Let $p, q$ be primes with Legendre symbol $\left(\frac{p}{q}\right)=1, q$ sufficiently larger than $p$. Then there exists a graph $X^{p, q}=(V, E)$ (we preserve the standard notation of these graphs) with the following properties:

1) $|V|=n=q\left(q^{2}-1\right) / 2$. (The vertex set of $X^{p, q}$ is the set of points of the projective linear group $\left.P S L_{2}(q)\right)$;

2) $X^{p, q}$ is $(p+1)$-regular;

3) $\operatorname{girth}\left(X^{p, q}\right) \geq 2 \log _{p} q$;

4) $\alpha\left(X^{p, q}\right) \leq \frac{2 \sqrt{p}}{p+1} n$

5) $\chi\left(X^{p, q}\right) \geq \frac{p+1}{2 \sqrt{p}}$.

Hence $X^{p, q}$ can be chosen as an example of graph $G_{k, l}$ with at most $k^{3 l}$ vertices.

This whole area is a source of many applications (and beautiful mathematics) which exceeds the scope of this paper. But in passing let us stress that no full analogy of graphs $X^{p, q}$ is known for hypergraphs (see for example recent [54]). In particular, no small explicit construction of hypergraphs is known. The best result here is the work of G. Kun [51], where he constructs hypergraphs $G_{p, k, l}$ with number of vertices bounded by a primitive recursive function of $p, k, l$. This construction uses a "twisted" product to reduce the number of short cycles in a constructed hypergraph. One proves that this is a polynomial process, yet randomized at each step.

A fully deterministic small (or even bounded by a tower function of bounded height) construction of hypergraphs $G_{p, k, l}$ is still an open problem. 
Admittedly, however the derandomization techniques and advances of theoretical computer science make the "constructive questions" less clear (and probably less important too) than they were in the 1960s.

\section{Random placement construction}

Here we present perhaps the simplest probabilistic proof of both Theorem 1 and 3 (however not Theorem 2). Surprisingly, this proof seems to be a little known. We need the following lemma [72].

Lemma 4 Fix $p \geq 3, l \geq 3$ positive integers. For any $\varepsilon>0$ there exist $n_{0}(p, l, \varepsilon)$ such that for every $n \geq n_{0}(p, l, \varepsilon)$ there exists a p-uniform hypergraph $H(n, p, l, \varepsilon)=(X, \mathcal{M})$ with the following properties:

1) $|X|=n$;

2) $|\mathcal{M}| \geq n^{1+\varepsilon}$;

3) $\operatorname{girth}(X, \mathcal{M}) \geq l$.

This (for graphs) is the easier part of Erdős proof of Theorem 2: one considers (for large $n$ ) random $k$-uniform hypergraph $(X, \mathcal{M}), X=\{1,2, \ldots, n\}$ with $m=\left\lceil 2 n^{1+\frac{1}{\varepsilon}}\right\rceil$ edges. It is easy to prove that for these values of $m$, the average number of edges in a cycle of length $<l$ is $o(n)$. Taking a witness $(X, \mathcal{M})$ of this inequality and by deleting the corresponding edges in short cycles we get the desired hypergraph $H(n, p, l, \varepsilon)$.

However simple this lemma has many consequences.

Proof of Theorem 3 ([72]):

Let $p, k, l$ be fixed. Put $P=(p-1)(k-1)+1$ and consider $H(n, P, l, \varepsilon)=$ $(X, \mathcal{M})$ as in Lemma 4 . Let $\mathcal{H}$ be the class of all $p$-uniform hypergraphs $(X, \mathcal{N})$ where every $M \in \mathcal{M}$ contains exactly one edge $N \in \mathcal{N}$ (i.e. we assume $\left|\mathcal{N} \cap\left(\begin{array}{c}M \\ p\end{array}\right)\right|=1$ for every $\left.M \in \mathcal{M}\right)$. Every $(X, \mathcal{N})$ obviously has girth $\geq l . \mathcal{H}$ is a large set: $|\mathcal{H}|=a^{n^{1+\varepsilon}}$ where $a=\left(\begin{array}{l}P \\ p\end{array}\right)$.

However, given a partition $\pi$ of $X$ by $(k-1)$ colors we have only at most $(a-1)^{n^{1+\varepsilon}}$ hypergraphs in $\mathcal{H}$ for which $\pi$ is a coloring of $(X, \mathcal{N})$ (with no monochromatic edge). Thus there are at most $(k-1)^{n}(a-1)^{n^{1+\varepsilon}}<a^{n^{1+\varepsilon}}=$ $|\mathcal{H}|$ hypergraphs in $\mathcal{H}$ with chromatic number $<k$. Thus there exists a witness for Theorem 3 . 
In this way the desired high chromatic large girth hypergraph is constructed by randomly replacing edges of $\mathcal{M}$ (i.e. $P$-tuples) with copies of a fixed hypergraph $H_{0}$. In this proof $H_{0}$ is a hypergraph with $P$ vertices containing a single edge ( $p$-tuple).

The above random placement construction is very flexible. Satisfaction of the difficult condition on the girth is inherited from $\mathcal{H}(n, p, l, \varepsilon)$ and the chromatic number follows by the above easy counting argument. We have tried to illustrate it by following figure:

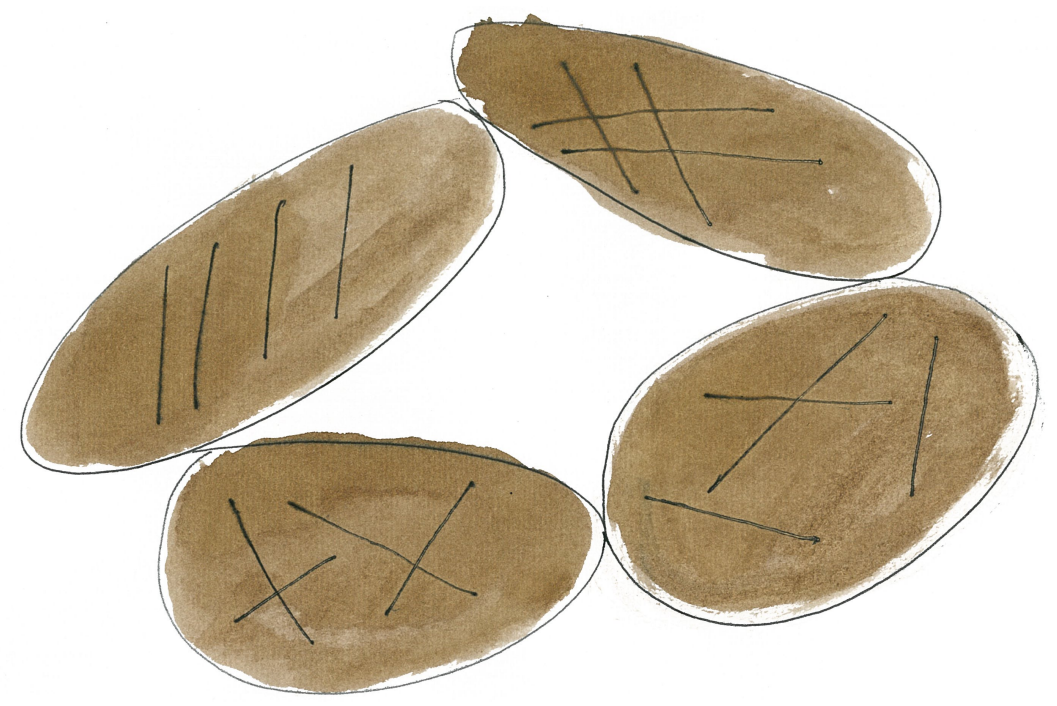

Yet another application is given in [72]. This is related to recent work on ergodic properties of topological subgroups of $S_{\omega}$. The combinatorial part of this development is motivated by the following definition which originated in structural Ramsey theory. We formulate it for graphs (for hypergraphs and, more generally, relational structures the definition and subsequent statements hold with little change).

An ordered graph $\vec{G}$ is a graph $G=(V, E)$ together with a linear ordering $\leq$ of $V$. We say that a graph $G^{\prime}=\left(V^{\prime}, E^{\prime}\right)$ has the ordering property for $\vec{G}$ if for any ordering $\preccurlyeq$ of $V^{\prime}$ (i.e. for any ordered graph $\vec{G}^{\prime}$ ) there exists an embedding $\varphi: G \rightarrow G^{\prime}$ which is monotone with respect to $\leq$ and $\preccurlyeq$.

For example, it is a classical result of graph theory that $\chi(G) \geq k$ if and only if $G$ has the ordering property for the monotone path with $k$ vertices (known as the Gallai-Hasse-Vitaver-Roy theorem).

We have the following:

Theorem 5 For every graph $G=(V, E)$ there exists a graph $G^{\prime}$ with the following properties: 
1) $G^{\prime}$ has the ordering property for any ordered graph $\vec{G}$;

2) if $\operatorname{girth}(G) \geq l$ then $\operatorname{girth}\left(G^{\prime}\right) \geq l$.

Proof. Given $\vec{G}$ with $p$ vertices, consider $H(n, p, l, \varepsilon)=(X, \mathcal{M})$ and consider all random placements $H$ of $G$ on edges of $\mathcal{M}$. Put $\frac{p !}{|\operatorname{Aut}(G)|}=a$ (this is the number of distinct placements of $G$ on a $p$-element set). The number of all graphs $H$ is thus $a^{|\mathcal{M}|}=a^{n^{1+\varepsilon}}$. However only at most $(a-1)^{n^{1+\varepsilon}} \cdot n$ ! do not have the ordering property for a $\vec{G}$. Thus there is a witness $G^{\prime}$ which has the ordering property for all $\vec{G}$.

In particular, for the cycle $C_{l}, l>3$, we obtain an undirected graph of girth $l$ which fails to be a cover graph of any partial order.

The existence of high girth non cover graph of posets (proved in [72]) led to the proof that the following recognition problem is NP-complete [78], [14]:

Input: A graph $G$.

Question: Is $G$ a cover graph of a finite poset?

The question was refined in [86] to lattices and this paper also contains a polynomial algorithm (using Ramanujan graphs) which constructs for given $k$ and $l$ a graph $G_{k, l}$ with $\operatorname{girth}\left(G_{k, l}\right)=l$ and $\chi\left(G_{k, l}\right)=k$ (see also [22]).

It is clear that every ordering of $G^{\prime}$ constructed in this way contains many copies of $\vec{G}$. Recently Angel, Kechris and Lyons [6] isolated in the interesting context of topological dynamics (characterizing structures with unique ergodic measure) the following property of a random placement graph $G^{\prime}$ : For graphs $G, G^{\prime}$ we denote by $\operatorname{emb}\left(G, G^{\prime}\right)$ the number of all embeddings of $G$ into $G^{\prime}$. Similarly emb $\left(\vec{G}, \vec{G}^{\prime}\right)$ denotes the number of monotone embeddings (with respect to orderings of $\vec{G}, \vec{G}^{\prime}$ ) of $G$ into $G^{\prime}$

Proposition 6 Let $\varepsilon>0$ be given. For every 2-connected graph $G$ there exists a graph $G^{\prime}$ such that for every pair of ordered graphs $\vec{G}$ and $\vec{G}^{\prime}$

$$
\left|\frac{e m b\left(\vec{G}, \vec{G}^{\prime}\right)}{e m b\left(G, G^{\prime}\right)}-\frac{1}{n !}\right|<\varepsilon .
$$

The proof follows again by letting $G^{\prime}$ be the random placement of copies of $G$ and applying Chernoff's inequality.

A generalization of Proposition 6 has been proven recently in [79], which further exploits the random placement construction to ordering property. Let us review it briefly. 
In this setting it is convenient to view orderings as permutations. Let $\sigma$ : $[n] \rightarrow[n]$ be a permutation of $[n]=\{1,2, \ldots, n\}$. For $X \subseteq[n]$ let $\sigma_{X}$ be the subpermutation of $\sigma$ induced by the set $X$ (i.e. if $X=\left\{i_{1}<i_{2}<\ldots<i_{k}\right\}$ then $\sigma_{X}(a)<\sigma_{X}(b)$ iff $\left.\sigma\left(i_{a}\right)<\sigma\left(i_{b}\right)\right)$.

Let $k \leq n$ (and typically $k$ is much smaller than $n$ ) and let $\pi_{1}, \ldots, \pi_{k}$ ! be a fixed enumeration of all permutations of $[k]$. The $k$-statistics of $\sigma$ is a sequence $s_{1}^{\sigma}, \ldots, s_{k !}^{\sigma}$ where $s_{i}^{\sigma}=\left|\left\{X \in\left(\begin{array}{c}{[n]} \\ k\end{array}\right) ; \sigma_{X}=\pi_{i}\right\}\right| /\left(\begin{array}{l}n \\ k\end{array}\right)$.

An ordered graph $\vec{G}$ on $[n]$ may be coded as $(G, \sigma)$ for a permutation $\sigma$ of $[n]$. We still call $(G, \sigma)$ an ordered graph (by permutation $\sigma$ ).

Let $\left(G^{\prime}, \sigma^{\prime}\right)$ be an ordered graph on $[N]$. An embedding $(G, \sigma)$ into $\left(G^{\prime}, \sigma^{\prime}\right)$ is a monotone injection $f:[n] \rightarrow[N]$ which is embedding of $G$ into $G^{\prime}$ and which satisfies

$$
\sigma(i)<\sigma(j) \text { if and only if } \sigma^{\prime}(f(i))<\sigma^{\prime}(f(j))
$$

Theorem 7 ([79]) Let $G$ be a 2-connected graph with $k$ vertices. Let $\vec{a}=$ $\left(a_{1}, \ldots a_{k !}\right)$ be a stochastic vector. Then for any $\varepsilon>0$ there exists a graph $H$ with $n$ vertices with the following properties

1) $\operatorname{girth}(G)=\operatorname{girth}(H)$;

2) if $\sigma$ is a permutation of $[n]$ with $k$-statistics $\left(s_{1}, s_{2}, \ldots, s_{k !}\right)$ then

$$
\left|\frac{e m b\left(\left(G, \pi_{l}\right),(H, \sigma)\right)}{\operatorname{emb}(G, H)}-b_{l}\right|<\varepsilon
$$

where

$$
b_{l}=\sum\left\{a_{i} s_{j}^{\sigma} ; \pi_{i} \circ \pi_{j}=\pi_{l}\right\} .
$$

It is easy to see that for the uniform probability $\vec{a}=(1 / k !, 1 / k !, \cdot, 1 / k !)$ we get Proposition 6 .

We also obtain the following "sparsification lemma", which is perhaps of independent interest

Lemma 8 For every $l, k \geq 2, \varepsilon>0$, there exists $n$ and $\mathcal{M} \subseteq\left(\begin{array}{c}{[n]} \\ k\end{array}\right)$ such that

1) $([n], \mathcal{M})$ has no cycles of length $\leq l$;

2) for every permutation $\sigma$ of $[n]$ it holds that $\left|s_{i}^{\sigma}-s_{i}^{\sigma}(\mathcal{M})\right|<\varepsilon$, where $s_{i}^{\sigma}(\mathcal{M})=\left|\left\{M \in \mathcal{M} ; \sigma_{M}=\pi_{i}\right\}\right| /|\mathcal{M}|$. 
Thus the $k$-statistics of every permutation $\sigma$ on $[n]$ are approximated by $k$-statistics on edges of $\mathcal{M}$ (and yet $\mathcal{M}$ has no short cycles).

The random placement construction has further applications to most coloring problems studied. For example it readily implies one of the main results of [91]. Other applications of random placement construction to coloring of graphs and hypergraphs are contained in [48], [45].

\section{Other voices, other rooms}

Coloring problems are among the most frequently studied combinatorial problems. One general approach is based on the notion of a homomorphism: Given graphs $G=(V, E)$ and $G^{\prime}=\left(V^{\prime}, E^{\prime}\right)$, a homomorphism is any mapping $f: V \rightarrow V^{\prime}$ which satisfies $\{x, y\} \in E \Rightarrow\{f(x), f(y)\} \in E^{\prime}$.

It is easy to see that $G$ has a homomorphism to a complete graph $K_{k}$ if and only if $\chi(G) \leq k$. Motivated by this, a homomorphism $G \rightarrow H$ is also called an $H$-coloring. Of course, if $G \not \rightarrow K_{k}$ then also $G \not \rightarrow H$ for every $H$ with $\chi(H) \leq k$. But as homomorphisms compose (i.e. form a category) we can prove stronger and (arguably more elegant) statements. These also indicate that sparsity is not a strong restriction in many coloring problems.

The main results proved in [83] may be formulated as follows. Because of its connection to rigid graphs and homomorphism order it is called sparse incomparability lemma (see e.g. [36]) and it holds for relational systems generally.

Theorem 9 For every graph $H$ and for all positive integers $k$ and $l$ there exists a graph $G$ with the following properties:

1) $\operatorname{girth}(G)>l$;

2) for every graph $F$ with at most $k$ vertices, there exists a homomorphism $g: G \rightarrow F$ if and only if there exists a homomorphism $f: H \rightarrow F$.

For the statement of the second result of [83] we need the following notion: A graph $F$ is said to be pointed for a graph $H$ (or shortly $H$-pointed) if any two distinct homomorphisms $H \longrightarrow F$ differ in at most 2 vertices. In other words this means that if two homomorphisms $g, g^{\prime}: H \rightarrow F$ satisfy $g(x)=g^{\prime}(x)$ for all $x \neq x^{\prime}$ (for some fixed vertex $x_{0} \in V(H)$ ) then it also holds that $g\left(x_{0}\right)=g^{\prime}\left(x_{0}\right)$. A graph $H$ is called a core if any homomorphism 
$H \rightarrow H$ is an automorphism. Note that any core graph $H$ is $H$-pointed and it follows that most graphs $H$ on a large set are $H$-pointed.

Theorem 10 For every graph $H$ and for every choice of positive integers $k$ and $l$ there exists a graph $G$ together with a surjective homomorphism $c: G \rightarrow H$ with the following properties:

1) $\operatorname{girth}(G)>l$;

2) for every graph $F$ with at most $k$ vertices, there exists a homomorphism $g: G \rightarrow F$ if and only if there exists a homomorphism $f: H \rightarrow F$;

3) for every $H$-pointed graph $F$ with at most $k$ vertices and for every homomorphism $g: G \rightarrow F$ there exists a unique homomorphism $f$ : $H \rightarrow F$ such that $g=f \circ c$.

Conditions 2) and 3) may be expressed by the following diagram:

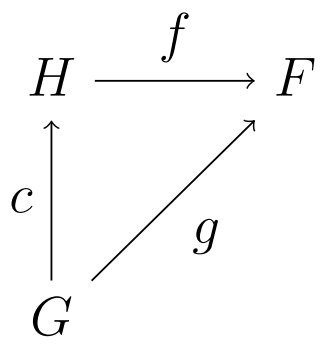

Theorem 10 may look like a technical extension of Theorem 9. However, it has several interestings corollaries from which we obtain approve structural extension of Erdős' Theorem 1.

A graph $G$ is uniquely $H$-colorable if there is a surjective homomorphism $c$ from $G$ onto $H$, and any other homomorphism from $G$ to $H$ is the composition $\sigma \circ c$ of $c$ with an automorphism $\sigma$ of $H$. (Note that this implies that $H$ is a core graph.)

The problem of the existence of uniquely $k$-colorable graphs with large girth has an interesting history: the triangle-free case (i.e. $l=3$ ) was settled in [68] and this was improved in [31] to graphs not containing short odd cycles. The general case was solved by Vladimír Müller [64,65]. Müller's proof is constructive and uses a constructive proof of Theorem 1. A nonconstructive proof has been published in [12] and the particular case $H=H^{\prime}$ of our Theorem 10 (i.e. the existence of uniquely $H$-colorable graph $G$ with 
girth $>l$ ) is proved in [91]. The above Theorem 10 then implies that there is a graph $G$ which is strongly uniquely $H$-colorable in the sense that any homomorphism $G \rightarrow F$ to any small $H$-pointed graph $F$ is induced by a homomorphism $H \rightarrow F$.

A probabilistic proof of Theorem 10 ([83]) is yet another variant of Erdős method and follows by a now standard pattern [51], [62]. Suppose $H$ has vertices $\{1,2, \ldots, a\}$ and let $H$ have $q$ edges. Let $V_{1}, \ldots, V_{a}$ be disjoint sets each of (large) size $n$. Let $G_{0}$ be the graph with vertex set $V=V_{1} \cup \ldots V_{a}$ and let $\{x, y\} \in E\left(G_{0}\right)$ if and only if $x \in V_{i}, y \in V_{j}$ and $\{i, j\} \in E(H)$. Let $\mathbb{G}$ be a random subgraph of $G_{0}$ with $q n^{1+\varepsilon}$ edges where $0<\varepsilon<1 / 4 l$. This may be viewed as we are replacing each vertex of $H$ by a large cloud (with $n$ vertices) and then taking a sparse random subgraph between clouds corresponding to edges of $H$. Theorem 9 then follows: If we have a coloring $c: V \rightarrow\left\{1, \ldots k^{\prime}\right\}, k^{\prime} \leq k$, then for each $i=1, \ldots, a$ let $V_{i}^{\prime} \subseteq V$ be the largest monochromatic subset and call the corresponding color $c(i)$. One then observes that if $\{i, h\} \in E(H)$ then $c(i) \neq c(j)$ because between $V_{i}^{\prime}$ and $V_{j}^{\prime}$ there have to be some edges. Thus if $c$ is a homomorphism $\mathbb{G} \rightarrow H^{\prime}$, $V(H)=\left\{1, \ldots, a^{\prime}\right\}$ then with high probability $c$ induces a homomorphism $H \rightarrow H^{\prime}$. The proof of part 3) of Theorem 10 needs more care as we have to treat small subsets, see [83].

In the style of this paper we add a schematic figure:
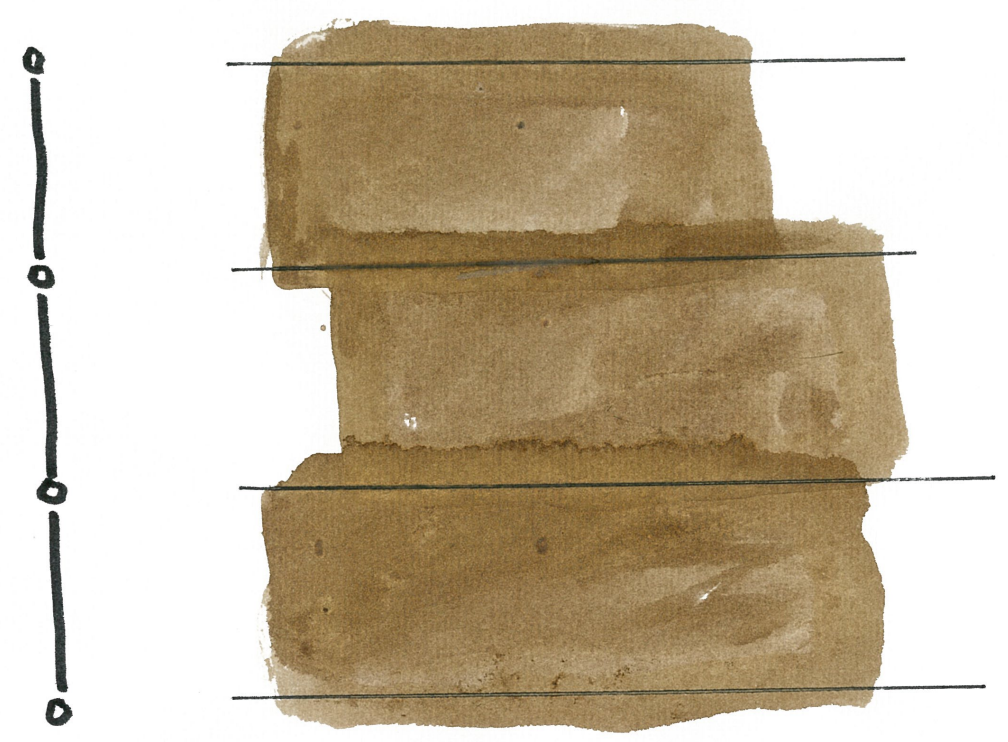

Note that this proof can be derandomized and an explicit (polynomial size) construction can be given (using Ramanujan graphs) [62]. Kun [51] gives a polynomial algorithm to construct set system (and more generally 
relational systems) satisfying 1), 2) of Theorem 10 . This allowed to close the hierarchy of descriptive complexity of classes defined and asked in [29]. In technical terms this amounts to MMSNP = CSP [51].

There is here more than meets the eye. One can prove also a strong extension of Müller's Extension Theorem [64, 65]. To do so we need another (this time categorical) notion:

A $t$-projective graph is a graph $G$ with the property that for every homomorphism (in this setting usually called polymorphism) $f: G \underbrace{\times \ldots \times}_{t} G \rightarrow G$ which satisfies $f(x, \ldots, x)=x$, there exists $i_{0}$ such that $f\left(x_{1}, x_{2}, \ldots, x_{t}\right)=$ $x_{i_{0}}$ (i.e. every idempotent homomorphism is a projection). It was proved in [53] that a graph is 2-projective if and only if it is $t$-projective for every $t$ (this is not true in general for relational structures).

The following result takes us from colorings to arbitrary $H$-colorings (and holds for general finite relational structures as well):

Corollary 11 Let $H$ be projective graph with $k$ vertices, and $l$ a positive integer. Let $A$ be a finite set and let $f_{1}, f_{2}, \cdots, f_{t}$ be distinct mappings $A \rightarrow$ $V(H)$. Then there exists a graph $G=(V, E)$ such that the followings hold:

1) $A$ is a subset of $V$;

2) for every $i=1,2, \cdots, t$ there exists unique homomorphism $g_{i}: G \rightarrow H$ such that $g_{i}$ restricted to the set $A$ coincides with the mapping $f_{i}$;

3) for every homomorphism $f: G \rightarrow H$ there exists $i, 1 \leq i \leq t$ and an automorphism $h$ of $H$ such that $h \circ f_{i}=f$;

4) G has girth $>l$.

Müller's Extension Theorem corresponds to $k$-colorings (i.e. $H=K_{k}$ ) which uniquely extend a given set partition and is depicted in the following figure: 


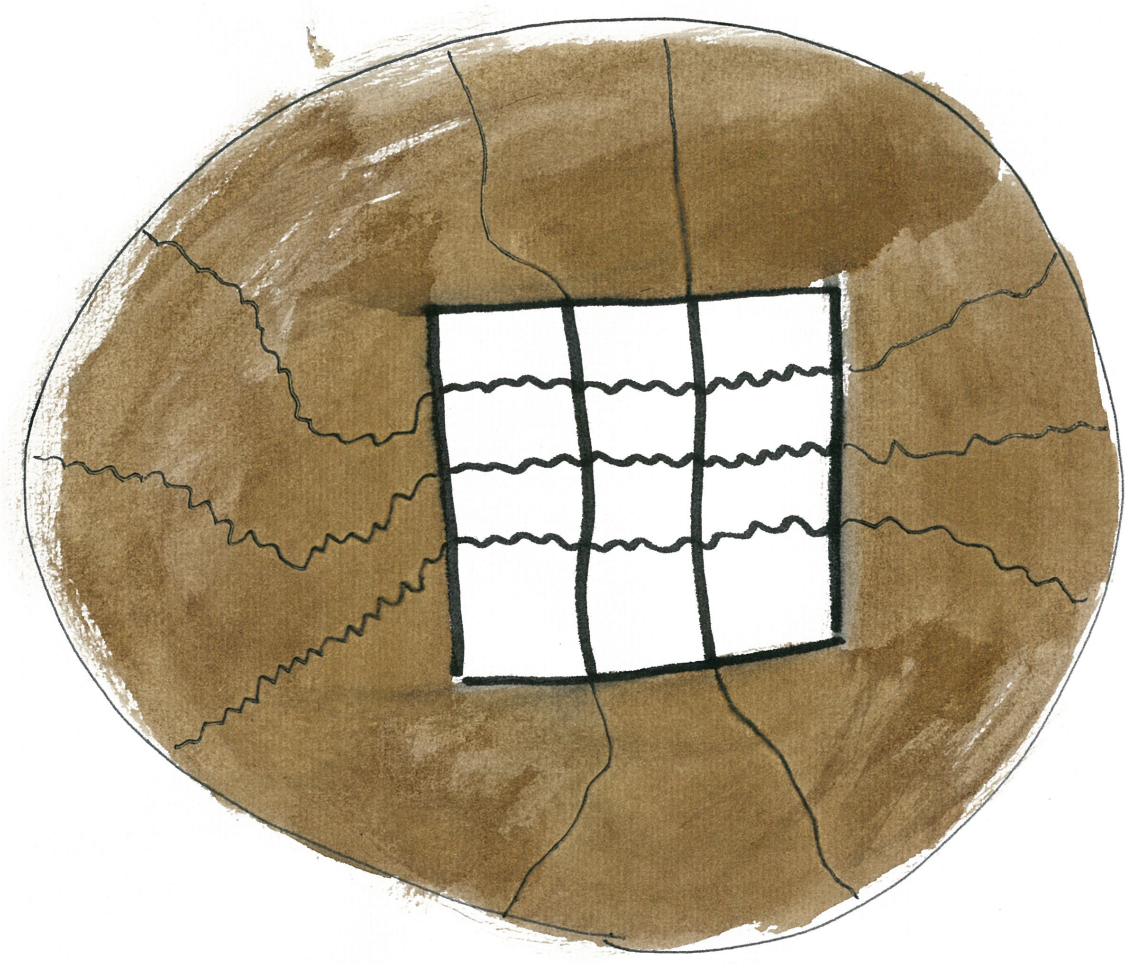

This is not just a generalization, this is as far as we can go:

Corollary 12 For a core graph $H$, the following statements are equivalent:

I. Corollary 11 holds.

II. The graph $H$ is projective.

These results and corresponding notions are not only interesting as an ultimative strengthening of Erdös' Theorem 1 in a more structural setting. In fact the above results hold for relational structures (or finite relational models). This is important for the complexity of algorithms, particularly in the context of Constraint Satisfaction Problems - CSP:

For a given a relational structure $\mathbf{H}, C S P(\mathbf{H})$ denotes the following decision problem:

Input: A structure A

Question: Does there exists a homomorphism $\mathbf{A} \rightarrow \mathbf{H}$.

It is conjectured [29] that this problem falls into just two classes: NP-Complete problems and polynomially solvable problems. This Dichotomy Conjecture [29] was investigated in the context of (universal) algebra [40], [15], [7], combinatorics and graph theory [36], threshold phenomena and random walks [52], for survey of this development see e.g. [36]. The dichotomy 
conjecture has then a refined form which conjectures an actual form of the dichotomy, see [37] Theorem 3.4. One of these formulations (using term "block projectivity") was isolated in [82]. Let us remark that recently the analogous question of dichotomy for counting homomorphisms (and CSP) was solved in the full generality in a major paper [16].

Theorem 11 plays an important role in this reduction (via so called fibre construction). It further follows from this result that the conjectured Dichotomy is very robust: it does not change if we restrict to objects with girth $\geq l$ and to structures with degree of its vertices bounded by $D(\mathbf{H})$. For this we need an effective version of Corollary 11 which is provided by $[62,51]$. As a particular case the following problem is NP-complete for any non-bipartite graph $H$ (and any fixed $l$ ):

Input: Graph $G$ with girth $\geq l$.

Question: Does there exists an $H$-coloring of $G$ ?

So after all the work the large girth restriction is not much of an obstacle.

\section{Limitations, perspectives and problems}

We can interpret negatively many of the above results: Despite the apparent difficulty high girth graphs with large chromatic number exist and their complexity and special properties seem not to be influenced by this (severe) restriction.

Here we add a few structural results which indicate opposite. We start with two infinite limitations.

\subsection{No universal $C_{4}$-free graph}

Theorem 13 ([33]) There is no countable graph of girth $>4$ which contains every countable graph of girth $>4$ as a subgraph.

In other words, there is no countable universal graph for the class of all graphs of girth $>4$. The same holds for the class of graphs with girth $>l \geq 4$. On the other hand, such a universal graph exists for the class of all triangle-free graphs. (The same is true, more generally, for the class of all graphs not containing short odd cycles [18].) In fact one can "forbid" homomorphisms from any finite set of graphs, see [17]. But this does not surprise an interested reader: odd cycles are easier in the whole paper. 


\subsection{No (transfinite) unbounded $\chi$}

Theorem 14 ([25]) Every graph $G$ with chromatic number $>\omega$ (i.e. of uncountable chromatic number) contains a complete bipartite subgraph $K_{\omega, n}$ for arbitrary finite $n$ and thus in particular the quadrangle $K_{2,2}$.

Thus the graphs $G_{k, l}$ are strictly finite objects which do not have, in full generality, an analogy in the infinite.

\subsection{Erdős-Hajnal}

Conjecture $7.1([24])$ For every $k, l$ there exists $f(k, l)$ such that any graph $G$ with $\chi(G) \geq f(k, l)$ contains a subgraph $G^{\prime}$ of girth $>l$ and $\chi\left(G^{\prime}\right) \geq k$.

This beautiful and (at least at first glance) plausible conjecture is in fact a very hard problem. The only known non-trivial case is that $f(k, 4)$ exists (Rödl [84]). (Note that Theorem 1 is equivalent to saying that Conjecture 7.1 holds for complete graphs.)

This conjecture appeared recently in the context of (homomorphism) restricted dualities, cf. [71, Chapter 11].

\subsection{Victor Neumann-Lara Conjecture}

Conjecture 7.2 There exists a function $g: \mathbb{N} \longrightarrow \mathbb{N}$ with the following property: Let $G=(V, E)$ be an undirected graph with $\chi(G) \geq g(k)$. Then there exists an orientation $\vec{G}=(V, \vec{E})$ of $G$ such that in every $k$-coloring of $V \vec{G}$ contains a monochromatic directed cycle.

There are many (perhaps too many) variants of chromatic number.

The minimal number of colors which suffice for coloring of vertices of digraphs so that no directed cycle is monochromatic is called (directed) acyclic chromatic number (thus the defining property is that the color classes induce acyclic subgraphs). Directed acyclic chromatic number was investigated and the analogy of Theorem 1 for directed acyclic coloring immediately follows from the random placement method (for a different proof see [9]). The existence of uniquely acyclic colorable was proved recently in [34]. 


\subsection{The Pentagon problem}

Conjecture 7.3 There exists an integer $l$ with the following property: If $G$ is a subcubic graph (i.e. every vertex has degree $\leq 3$ ) with girth $\geq l$ then $G \rightarrow C_{5}$.

Brook's theorem implies that every subcubic graph not containing $K_{4}$ satisfies $G \rightarrow C_{3}$. On the other hand this problem has a negative solution for $C_{11}$ [47], $C_{9}$ [90], and finally $C_{7}$ [35].

It is not even known whether high girth subcubic graphs have circular chromatic number $<3$.

\subsection{No Ramsey classes with girth $>3$}

A class $\mathcal{C}$ of graphs (or, more generally, structures) is said to be Ramsey if the following holds ([80], [81], [70]): For every $\mathbf{A}, \mathbf{B} \in \mathcal{C}$ and positive integer $k$ there exists $\mathbf{C} \in \mathcal{C}$ such that $\mathbf{C} \rightarrow(\mathbf{B})_{k}^{A}$ where (Erdős-Rado) partition arrow has the following meaning:

For every partition $\left(\begin{array}{l}\mathbf{C} \\ \mathbf{A}\end{array}\right)=\mathcal{A}_{1} \cup \ldots \mathcal{A}_{k}$ there exists $\mathbf{B}^{\prime} \in\left(\begin{array}{l}\mathbf{C} \\ \mathbf{B}\end{array}\right)$ such that $\left(\begin{array}{c}\mathbf{B}^{\prime} \\ \mathbf{A}\end{array}\right) \subseteq \mathcal{A}_{i}$ for some $i \in\{1, \ldots, k\}$. Here $\left(\begin{array}{l}\mathbf{C} \\ \mathbf{A}\end{array}\right)$ denotes the set of all substructures of $\mathbf{C}$ which are isomorphic to $\mathbf{A}$.

Ramsey's theorem claims that the class of complete graphs is a Ramsey class.

Theorem $15([69])$ The class $\mathcal{C}_{l}$ of all ordered graphs with girth $\geq l>4$ fails to be a Ramsey class.

This follows from [69] where the connection between Ramsey classes and ultrahomogeneous structures is isolated and as a result of this all Ramsey classes of graphs are determined (or better: it is shown that we know them all). This line of research was studied later intensively, see e.g. the important paper [42], and connections to extreme amenability of subgroups of $S_{\omega}$ were established.

\subsection{Edge Ramsey for large girth}

Problem 16 Does the class $\mathcal{C}_{l}$ have the edge-Ramsey property? 
Explicitly: Is it true that for every $G \in \mathcal{C}_{l}$ there exists $H=(V, E) \in \mathcal{C}_{l}$ such that for every partition $E_{1} \cup E_{2}$ of $E$ there exists a subgraph $G^{\prime}$ of $H, G^{\prime}$ isomorphic to $G$ such that $E\left(G^{\prime}\right)$ is a subset of either $E_{1}$ or $E_{2}$ (i.e. $H \rightarrow(G)_{2}^{K_{2}}$ in the above notation). This is known to be true for $l \leq 6$ [65]. This problem (together with Pisier type problems [26]) is one of the few that remained open in structural Ramsey theory.

\subsection{Persistence of Old Motivations.}

Many classical problems in this area still have no satisfactory answer. Particularly, the basic challenge in this area of complex large girth graphs is to find new constructions. The old questions remain. The recent advances of theoretical computer science put these problems in a new context and make these questions very actual.

Acknowledgment: I thank to Martin Bálek and Andrew Goodall for the help when preparing this article.

\section{References}

[1] M. Ajtai, J. Komlós, E. Szemerédi, A note on Ramsey numbers, J. Combi. Th. Series A, 29 (1980), 354-360.

[2] M. Ajtai, J. Komlós, E. Szemerédi, An $O(n \log n)$ sorting network, Proceedings of the 15th Annual ACM Symposium on Theory of Computing, 1983, 1-9.

[3] N. Alon, Discrete Mathematics: methods and challenges, Proc. of the International Congress of Mathematicians (ICM), Beijing 2002, China, Higher Education Press (2003), 119-135.

[4] N. Alon, V. Rödl, Sharp bounds for some multicolor Ramsey numbers, Combinatorica 25 (2005), 125-141.

[5] N. Alon, J. H. Spencer, The probabilistic method Second ed. WileyInterscience Series in Discrete Mathematics and Optimization, WileyInterscience, 2000. 
[6] O. Angel, A. Kechris, R. Lyons, Random Orderings and Unique Ergodicity of Automorphism Groups, arXiv: 1208.2389 (2012).

[7] L. Barto, M. Kozik, New conditions for Taylor varieties and CSP, In: Proceedings of LICS'10, IEEE, 2010, 100-109.

[8] S. Baum, M. Stiebitz, Coloring Of Graphs Without Short Odd Paths Between Vertices Of The Same Color Class, (preprint, TU Ilmenau).

[9] D. Bokal, G. Fijavž, M. Juvan, P. M. Kayll, B. Mohar, The circular chromatic number of a digraph, J. Graph Theory 46(2004), no. 3, 227240.

[10] B. Bollobás, Random Graphs, Academic Press, 1985.

[11] B. Bollobás, Modern Graph Theory, Springer-Verlag, 1998.

[12] B. Bollobás and N. Sauer, Uniquely colorable graphs with large girth, Can. J. Math. 28(1976), 1340-1344.

[13] J. A. Bondy, U. S. R. Murty, Graph theory, Graduate Texts in Mathematics, 244, Springer, 2008.

[14] G. Brightwell, On the complexity of diagram testing, Order 10,4 (1983), 297-303.

[15] A. A. Bulatov, P. Jeavons, A. A. Krokhin, Classifying the Complexity of Constraints Using Finite Algebras, SIAM J. Comput. 34(3), 2005, 720-742.

[16] J.-Y. Cai, X. Chen, P. Lu, Graph Homomorphisms with Complex Values: A Dichotomy Theorem, arXiv: 0903.4728v2.

[17] G. Cherlin, S. Shelah, N. Shi, Universal graphs with forbidden subgraphs and algebraic closure, Adv. in Applied Math. 22 (1999), 454-491.

[18] G. Cherlin, N. Shi, Graphs omitting a finite set of cycles, J. of Graph Th. 21 (1997), 351-355.

[19] B. Codenotti, P. Pudlák, J. Resta, Some structural properties of low rank matrices related to computational complexity, Theoretical Computer Sci. 235 (2000), 89-107. 
[20] B. Descartes, A three colour problem, Eureka 21, 1947.

[21] R. Diestel, Graph theory, Graduate Texts in Mathematics, 173, Springer, Heidelberg, 2010.

[22] T. Emden-Weinert, S. Hongardy, B. Kreutzer, Uniquelly colorable graphs and hardness of colouring of graphs of large girth, Comb. Prob. Comp. 7,4 (1998), 375-386.

[23] P. Erdős, Graph theory and probability, Canad. J. Math. 11 (1959), 3438.

[24] P. Erdős, Problems and results in combinatorial analysis and graph theory, In: Proof Techniques in Graph Theory, Academic Press, 1969, 2735 .

[25] P. Erdős, A. Hajnal, On chromatic number of graphs and set-systems, Acta Math. Acad. Sci. Hungar. 17 (1966), 61-99.

[26] P. Erdős, J. Nešetřil, V. Rödl On Pisier type problems and results (combinatorial applications to number theory), In: Mathematics of Ramsey Theory, Springer 1990, 214-231.

[27] P. Erdős, C. A. Rogers, The construction of certain graphs, Canad. J. Math. 14 (1962), 702-707.

[28] P. Erdős, E. Specker, On a theorem in the theory of relations and a solution of a problem of Knaster, Colloq. Math. 8 (1961), 19-21.

[29] T. Feder, M. Y. Vardi, The computational structure of monotone monadic SNP and constraint satisfaction: A study through Datalog and group theory, SIAM J. Comput. 28, 1 (1999), 57-104.

[30] R. Graham and B. Rothschild and J. Spencer, Ramsey Theory, Wiley, New York, 1990.

[31] D. Greenwell and L. Lovász, Applications of product coloring, Acta Math. Acad. Sci. Hungar. 25(1974), 335-340.

[32] A. Gyárfás, T. Jensen, M. Stiebitz, On graphs with strongly independent colour-classes, JGT 46 (2004), 1-14. 
[33] A. Hajnal, J. Pach, Monochromatic paths in infinite graphs, In: Finite and Infinite Sets, Coll. Math. Soc. J. Bolyai, Eger, 1981, 359-369.

[34] A. Harutyunyan, P. M. Kayll, B. Mohar, L. Rafferty, Uniquely Dcolourable Digraphs with Large Girth, Canad. J. Math. Vol. 64 (6), 2012, $1310-1328$.

[35] H. Hatami, Random cubic graphs are not homomorphic to the cycle of size 7, J. Comb. Th. B 93 (2005), 319-325.

[36] P. Hell, J. Nešetřil, Graphs and homomorphisms, Oxford University Press, 2004.

[37] P. Hell, J. Nešetřil, Colouring, Constraint Satisfaction, and Complexity, Comp. Sci. Review 2,3 (2008) 134-164.

[38] S. Hoory, N. Linial, A. Widgerson, Expander graphs and their applications, Bulletin (New series) of the American Mathematical Society 43 (4), 2006, 439-561.

[39] S. Janson, T. Luczak, A. Ruczinski, Random Graphs, Wiley, 2000.

[40] P. Jeavons, On the algebraic structure of combinatorial problems, Theor. Comp. Sci 200 (1998), 185-204.

[41] J. Kahn, Recent results on some not-so-recent hypergraph matching and covering problems, Proc. 1st Int'l Conference on Extremal Problems for Finite Sets, Visegrad, 1991.

[42] A. Kechris, Pestov, S. Todorcevic, Fraïssé limits, Ramsey theory and topological dynamics of automorphism groups, Geometrical and Functional Analysis 15(1), 2005, 106-189.

[43] J. Kelly and L. Kelly, Path and circuits in critical graphs, Amer. J. Math. 76:786-792, 1954.

[44] J. H. Kim, The Ramsey Number $R(3, t)$ has order of magnitude $t^{2} / \log t$, Random Structures and Algorithms 7 (1995), 173-207.

[45] A. V. Kostochka, D. Mubayi, J. Verstraete, Hypergraph Ramsey Numbers: Triangles versus Cliques, submitted. 
[46] A. V. Kostochka J. Nešetřil, Properties of Descartes' construction of triangle-free graphs with high chromatic number, Combin. Probab. Comput. 8(1999), no. 5, 467-472.

[47] A. Kostochka, J. Nešetřil, P. Smolíková, Coloring and homomorphism of degenerate and bounded degree graphs, Discrete Math. 233, 1-3 (2001), 257-276.

[48] A. V. Kostochka, V. Rödl, Constructions of Sparse Uniform Hypergraphs with High Chromatic Number, Random tructures and Algorithms, 2009, 46-56.

[49] I. Kříž, A hypergraph free construction of highly chromatic graphs without short cycles, Combinatorica 9 (1989), 227-229.

[50] I. Kříž, J. Nešetřil, Chromatic number of the Hasse diagrams, eyebrows and dimension, Order 8/1991/, 41 - 48.

[51] G. Kun, Constraints, MMSNP and expander relational structures, arXiv: 0706.1701 (2007).

[52] G. Kun, M. Szegedy, A new line of attack on the dichotomy conjecture, STOC 2009, 725-734. See also Electronic Coll. on Comp. Compl. (ECCC) 16:59 (2009), 44p.

[53] B. Larose, C. Tardif, Graph coloring problems, Wiley, 1995.

[54] J. Lenz, D. Mubayi, The poset of hypergraph quasirandomness, The Poset of Hypergraph Quasirandomness, arXiv:1208.5978 [math.CO] 29 Aug 2012.

[55] L. Lovász, On chromatic number of finite set-systems, Acta Math. Acad. Sci. Hungar. 19(1968), 59-67.

[56] L. Lovász, Kneser's conjecture, chromatic number, and homotopy, J. Combin. Theory. Ser. A 25 (1978), 319-324.

[57] L. Lovász, Combinatorial Problems and Exercises, Akad. Kiad, Budapest, 1979.

[58] A. Lubotzky, R. Phillips, P. Sarnak, Ramanujan graphs, Combinatorica, 8(3), 1988, 261-277. 
[59] M. Mareš, The saga of minimum spanning trees, Comp. Sci. Review 2 (2008), 165-221.

[60] G. A. Margulis, Explicit constructions of graphs without short cycles and low density codes, Problemy Pereači Informacii, 9(4), 1973, 71-80.

[61] J. Matoušek, Using the Borsuk-Ulam theorem, Lectures on topological methods in combinatorics and geometry, Springer, 2003.

[62] J. Matoušek, J. Nešetřil, Constructions of sparse graphs with given homomorphisms, (manuscript).

[63] M. Molloy, B. Reed, Graph colouring and the probabilistic method, Algorithms and Combinatorics, 23, Springer, 2002.

[64] V. Müller, On colorable critical and uniquely colorable critical graphs, in: Recent Advances in Graph Theory (ed. M. Hiedler), Academia, Prague, 1975.

[65] V. Müller, On coloring of graphs without short cycles, Discrete Math., 26(1979), 165-179.

[66] J. Mycielski, Sur le coloriage des graphes, Colloq. Math. 3, 161-162, 1955.

[67] J. Nešetřil, $K$-chromatic graphs without cycles of length $\leq 7$, (in Russian), Comment. Math. Univ. Carol., 7, 3(1966), 373-376.

[68] J. Nešetřil, On uniquely colorable graphs without short cycles, Časopis Pěst. Mat. 98(1973), 122-125.

[69] J. Nešetřil, For graphs there are only four types of hereditary Ramsey classes, J. Combin. Theory Ser. B 46, (1989), no. 2, 127-132.

[70] J. Nešetřil, Ramsey Theory, In: Handbook of Combinatorics (ed. R. L. Graham, M. Grötschel, L. Lovász), North-Holland, 1995, 1331-1403.

[71] J. Nešetřil, P. Osssona de Mendez, Sparsity - Graph, Structures, and Algorithms, Springer, 2012.

[72] J. Nešetřil, V. Rödl, On a probabilistic graph-theoretic Method, Proc. Amer. Math. Soc. 72 (1978), 417-421. 
[73] J. Nešetřil, V. Rödl, A short proof of the existence of restricted Ramsey graphs by means of a partite construction, Combinatorica 1,2 (1982), 199-202.

[74] J. Nešetřil, V. Rödl, Sparse Ramsey graphs, Combinatorica 4, 1 (1984), $71-78$.

[75] J. Nešetřil, V. Rödl, Combinatorial partitions of finite posets and lattices - Ramsey lattices, Algebra Univ. 19 (1984), 106-119

[76] J. Nešetřil, V. Rödl, Complexity of diagrams, Order 3 (1987), 321-330.

[77] J. Nešetřil, V. Rödl, Chromatically optimal rigid graphs, J. Combin. Th.(B), 46(1989), 133-141.

[78] J. Nešetřil, V. Rödl, More on complexity of diagrams, Comm. Math. Univ. Carol. 36,2 (1995), 269-278.

[79] J. Nešetřil, V. Rödl, Statistics of Orderings, (to appear).

[80] J. Nešetřil, V. Rödl, Partitions of Finite Relational and Set Systems, J. Comb. Th. A 22,3 (1977), 289-312.

[81] J. Nešetřil, V. Rödl, Partition (Ramsey) Theory - a survey, In: Coll. Math. Soc. János Bolyai, 18. Combinatorics, Keszthely 1976, North Holland, 1978, 759-792.

[82] J. Nešetřil, M. Siggers, L. Zadori, A Combinatorial Constraint Satisfaction Problem Dichotomy Classication Conjecture, European J. Comb. 31 (1), 2010, 280-296.

[83] J. Nešetřil, X. Zhu, On sparse graphs with given colorings and homomorphisms, J. Combin. Theory Ser. B 90(2004), no. 1, 161-172.

[84] V. Rödl, On the chromatic number of subgraphs of a given graph, Proc. Amer. Math. Soc. 64 (1977), 370-371.

[85] V. Rödl, On a packing and covering problem, Europ. J. Combinatorics 5 (1985), 69-78.

[86] V. Rödl, L. Thoma, The complexity of cover graph recognition for some vertices of finite lattices, Order 12,4 (1995), 351-374. 
[87] G. Simonyi and G. Tardos, Local chromatic number, Ky Fan's theorem, and circular colorings, Combinatorica 26 (2006), 587-620.

[88] J. Spencer, Ten Lectures on the Probabilistic Method, Society for Industrial and Applied Mathematics, 1987.

[89] W. T. Trotter, R. Wang, Incidence Posets and Cover Graphs, to appear in Order.

[90] I. M. Wanless, N. C. Wormald, Regular graphs with no homomorphisms onto cycles, J. Comb. Th. B 82 (2001), 155-160.

[91] X. Zhu, Uniquely H-colorable graphs with large girth, J. Graph Theory, 23 (1996), 33-41.

[92] A. A. Zykov, On some properties of linear complexes, Mat. Sbornik 24:313-319, 1949. (In Russian). 